\title{
Pseudomonas aeruginosa Takes a Multi-Target Approach to Achieve Junction Breach
}

\author{
Guillaume Golovkine ${ }^{\dagger}$, Emeline Reboud and Philippe Huber* \\ Centre National de la Recherche Scientifique ERL5261, CEA BIG-BCI, Institut National de la Santé et de la Recherche \\ Médicale UMR1036, Université Grenoble Alpes, Grenoble, France
}

Pseudomonas aeruginosa is an opportunistic pathogen which uses a number of strategies to cross epithelial and endothelial barriers at cell-cell junctions. In this review, we describe how the coordinated actions of $P$. aeruginosa's virulence factors trigger various molecular mechanisms to disarm the junctional gate responsible for tissue integrity.

Keywords: bacterial invasion, bacterial virulence factors, intercellular junctions, bacterial secretion systems, epithelium, endothelium

\section{OPEN ACCESS INTRODUCTION}

Edited by:

Matthew C. Wolfgang,

University of North Carolina at Chapel Hill, United States

Reviewed by:

Michael L. Vasil,

University of Colorado Denver School of Medicine, United States

Gregory Anderson,

Indiana University, Purdue University Indianapolis, United States

*Correspondence:

Philippe Huber

phuber@cea.fr

${ }^{\dagger}$ Present Address:

Guillaume Golovkine,

Department of Molecular and Cell

Biology, University of California,

Berkeley, Berkeley, CA, United States

Received: 07 November 2017 Accepted: 20 December 2017

Published: 11 January 2018

Citation:

Golovkine G, Reboud E and Huber P (2018) Pseudomonas aeruginosa Takes a Multi-Target Approach to Achieve Junction Breach. Front. Cell. Infect. Microbiol. 7:532. doi: 10.3389/fcimb.2017.00532
Transmigration across the body's natural barriers is one of the most significant challenges for bacterial pathogens. This process is particularly challenging for opportunistic pathogens, which tend to be less armed than true pathogens. Once the bacterium has penetrated the host's external defenses it can exploit the many advantages: abundant nutrients, replicative niche and lack of bacterial competition. Although the blood is a very bactericidal environment, it can be transiently used by bacteria after entry into the body as a route to spread and thus reach the retro-endothelial compartment of many organs.

In this review, we will focus on a major opportunistic Gram-negative pathogen, Pseudomonas aeruginosa, responsible for nosocomial acute and chronic infections, in particular in patients with cystic fibrosis (Williams et al., 2010; Gellatly and Hancock, 2013). In chronic infections, $P$. aeruginosa colonizes the airways and forms biofilms where multiple bacteria proliferate. In acute infections, $P$. aeruginosa traverses the mucosa, provoking bacteremia and infecting distant organs.

Unless there is a breach, the skin and most multilayered mucosae (mouth, esophagus, vagina, etc.) are impenetrable to opportunistic pathogens like $P$. aeruginosa. The gut epithelium, with its extensive mucus barrier and highly competitive microbiota is also very difficult to traverse. For these reasons, bacteria mostly transmigrate at the level of the lung alveoli and the urinary tract, composed of a single layer or transitional epithelia, as well as the cornea, composed of a small number of non-keratinized cell layers (Lyczak et al., 2000; Mittal et al., 2009; Fleiszig and Evans, 2010; Williams et al., 2010). Once present in the interstitium, bacteria may reach the blood by crossing the endothelium, using specific mechanisms that will be discussed in this review.

Several mechanisms have been described by which bacterial pathogens cross the epithelium, including transcellular and paracellular (i.e., through intercellular junctions) routes and a Trojan horse strategy, where phagocytic cells are used as transporters (Doran et al., 2013). Here, we will focus on the mechanisms by which $P$. aeruginosa has been shown to traverse the epithelial and endothelial barriers.

Abbreviations: 3O-C12-HSL, N-(3-oxododecanoyl)-L-homoserine lactone; AJ, adherens junction; QS, quorum sensing; T2SS, Type II secretion system; T3SS, Type III secretion system; TJ, tight junction. 
$P$. aeruginosa is mainly an extracellular bacterium, although several groups have reported its possible internalization in nonimmune cells (i.e., various epithelial cell types) in vitro (Fleiszig et al., 1994; Hauser et al., 1998; Kierbel et al., 2005; Zaas et al., 2009; Sana et al., 2015). However, it is not currently known whether $P$. aeruginosa is capable of traversing the epithelial layer once internalized, and no Trojan horse transmigration events have been documented so far. Several old and more recent works describe how $P$. aeruginosa alters intercellular junction components to allow its transmigration at cell-cell junctions. This body of literature suggests that the paracellular route is the main itinerary used by this bacterium to gain entry to the host (Zulianello et al., 2006; Heiniger et al., 2010; Golovkine et al., 2016a). However, before they even reach the junctions, bacteria must avoid a number of general defense mechanisms.

\section{THE MUCOSAE AND THEIR NUMEROUS DEFENSE MECHANISMS}

The apical side of all mucosae are coated by a liquid layer of variable viscosity: mucus, surfactant, tears or urine. This liquid layer entraps bacteria, and flows continuously to prevent bacterial adhesion to surfaces while clearing bacteria out of the body. In the specific case of the lung, where gravity cannot assist bacterial expulsion, the muco-ciliary escalator efficiently removes bacteria from the airways. This system moves the mucus blanket in the direction of the larynx, by a mechanism promoted by the beating of cilia present on tracheal cells. In addition to the physical effects of this fluid layer surmounting epithelia, it generally contains host molecules with antimicrobial activity, such as defensins and lysozyme, as a complement to the first line of anti-bacterial defense (Williams et al., 2010; Gellatly and Hancock, 2013).

When bacteria come into contact with mucosae, a second line of defense is triggered, involving the production of proinflammatory cytokines and chemokines, followed by the massive recruitment of neutrophils which can internalize and eliminate bacteria thanks to phagocytic processes. LPS, flagellum, and type IV pili (pili hereafter) are efficient activators of this defense system (Williams et al., 2010).

If, despite these defensive strategies, bacteria nevertheless reach epithelial cells, two epithelial defense mechanisms further impede bacterial transmigration:

(1) The epithelial cell apical domain is refractive to toxin injection by the type III secretion system (T3SS) (Fleiszig et al., 1997; Lee et al., 1999; Kazmierczak et al., 2004), one of $P$. aeruginosa's most potent virulence factors (see below).

(2) The integrity of the epithelial barrier is ensured by several junctional structures located in the intercellular space (Farquhar and Palade, 1963; Cereijido et al., 2004); these structures are described below (Figure 1).

Tight junctions (TJs), located near the apical side of the epithelium, are composed of a branching network of close contacts between neighboring cells, forming a continuous junction ("zonula") at the cellular periphery and establishing a "gate" between the apical and basal compartments. The transmembrane proteins mediating homophilic adhesion at TJ

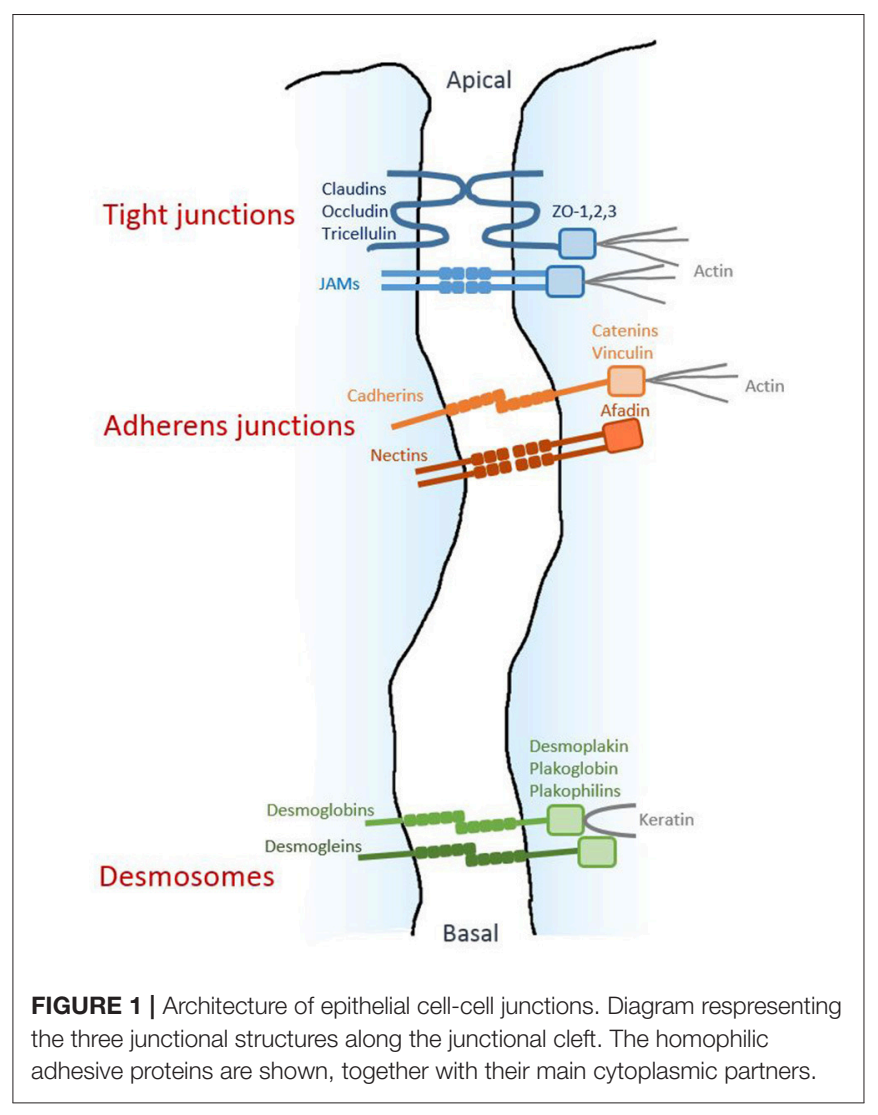

are members of the claudin family, as well as occludin, tricellulin and the junctional adhesion molecule (JAM) proteins. Their intracellular domains interact with ZO-1, -2 and -3 proteins, which constitute molecular platforms for various cytosolic components, including actin filaments.

The adherens junctions (AJs), located more basally, also form a zonula mainly composed of E-cadherin transmembrane proteins clustering in cis and promoting homophilic adhesion in trans with E-cadherin molecules on adjacent cells. Inside the cell, these junctions are linked to the actin cytoskeleton by catenins and vinculin. The adhesive activity of the AJ complex is finetuned by tyrosine- and serine/threonine-kinases, as well as Rac and Rap1 GTPases (Boettner and Van Aelst, 2009; Serrels et al., 2011; McCole, 2013; Ratheesh et al., 2013). Nectin is another transmembrane protein with homophilic adhesive activity; it is located in AJs and is linked to actin by afadin. Importantly, the presence of AJs is required for the formation and maintenance of the other junctional structures.

Finally, closer to the basal side of the epithelium, the desmosomes form spots ("macula") along the cell's circumference. These macula are composed of adhesive proteins desmoglein and desmoglobin, which are linked to keratin filaments by desmoplakin, plakoglobin, and plakophilins.

While both TJs and AJs are targets of $P$. aeruginosa's virulence factors (see below), so far, no toxic action of $P$. aeruginosa on desmosomes has been reported.

All of these multi-bolt junctional structures work together to provide strong interactions between epithelial cells and 
strictly control paracellular permeability to molecules and cells.

In normal settings, epithelia are thus protected from bacterial infiltration by an array of mechanisms. However, after certain physical, chemical or biological insults, the priming effects of which have yet to be completely characterized in vivo, mucosae can become permissive to bacteria.

\section{INITIAL STAGES OF P. AERUGINOSA-MUCOSA INTERACTION}

Before penetration, bacteria must first reach a site compatible with transmigration. A polar flagellum is present in most P. aeruginosa strains. This structure allows the bacteria to swim in liquids and rapidly scan cellular surfaces for transmigration opportunities (Luzar et al., 1985). In this context, $P$. aeruginosa investigates the cell surface by employing several types of swimming trajectories (Golovkine et al., 2016b). Recent findings indicate that an active flagellum is indeed required for efficient infection in vivo (Turner et al., 2014).

Another type of motion is provided by pili. Pili are thin filaments which extend and retract through filament assembly and disassembly in bacterium's inner membrane (Leighton et al., 2015). The tip of the pilus displays adhesive properties allowing bacteria to interact with abiotic and cellular surfaces. By retracting attached pili and detaching others, $P$. aeruginosa can engage in a type of surface motility by twitching movement.

Both swimming and twitching motions allow bacteria to investigate the mucosa and to resist the host's flushing systems.

Bacteria bound to the epithelial surface can also remain immobile and form aggregates of tens to hundreds of individuals embedded in a biofilm-like matrix (Lepanto et al., 2011). This bacterial aggregate can locally alter the apical surface of the host cell, transforming it into an area with basolateral-domain composition (Kierbel et al., 2007). The transformed area can then recruit AJ proteins, like E-cadherin/ $\beta$-catenin and afadin. A similar process has been reported for Neisseria meningitidis at the apical surface of the blood-brain barrier, which eventually leads to junction attrition (Coureuil et al., 2009). However, for $P$. aeruginosa, this process does not disrupt intercellular junctions (Kierbel et al., 2007; Tran et al., 2014).

As mentioned above, studies with in vitro and ex vivo epithelial systems converge toward a mechanism in which bacterial transmigration occurs at cell-cell junctions. However, not all junctions are permissive to bacterial transmigration; transmigration therefore remains a rare event in reconstituted polarized epithelia, and probably in vivo as well. Our group recently showed that some $P$. aeruginosa strains take advantage of naturally occurring mini-breaches in epithelial cell-cell junctions, either during cell division or when senescent cells are expelled from the epithelial layer (Figure 2A) (Golovkine et al., 2016a). In both situations, a transient loss of E-cadherin at cellcell junctions allows infiltration of one bacterium, followed by a cohort of other bacteria attracted by chemotaxis to the same entry-point. Therefore, tissue repair mechanisms may increase the capacity of $P$. aeruginosa to cross the epithelial layer.

\section{BACTERIAL VIRULENCE FACTORS INVOLVED IN JUNCTION DISRUPTION}

P. aeruginosa synthesizes a number of regulated virulence factors which work synergistically in the context of infection. In this review, we focus on factors that have been demonstrated to play a pivotal role in bacterial transmigration across epithelial and endothelial barriers. Their various actions on junctions are summarized in Table 1. Further information about $P$. aeruginosa virulence factors can be found in several excellent reviews (Hauser, 2009; Bleves et al., 2010; Sawa, 2014).

\section{LasB Protease}

LasB is a potent protease with broad substrate specificity. It is secreted into the extracellular medium by most $P$. aeruginosa strains through their T2SS (Bleves et al., 2010). LasB increases epithelial monolayer permeability and induces junction disruption by downregulating the expression of several TJ components: occludin, claudin-1 and-4, and tricellulin (Azghani et al., 1993, 2000; Beaufort et al., 2013; Nomura et al., 2014), without affecting E-cadherin in AJ (Azghani et al., 1993; Golovkine et al., 2014; Nomura et al., 2014). In response to LasB, decreased levels of ZO-1 and-2 have also been noted in the MDCK epithelial cell line, whereas no difference in their expression level was detected in nasal epithelial cells grown ex vivo (Azghani, 1996; Nomura et al., 2014). Several signaling proteins are involved in the transient downregulation of $\mathrm{TJ}$ protein expression, including protein kinase $\mathrm{C}$, the MAP kinases (ERK, P38 and JNK), phosphatidylinositol 3-kinase, the cyclooxygenase- 1 and -2 and the protease activated receptor-2 (Clark et al., 2011; Nomura et al., 2014). However, how these signaling proteins are altered by LasB is essentially unknown and their interplay with the TJ components remain elusive.

\section{Quorum Sensing}

The expression of LasB and several other bacterial proteins is controlled by quorum sensing (QS), a system of bacterial communication based on cell density (Schuster et al., 2003). One of the QS signaling molecules produced by bacteria, $\mathrm{N}-(3-$ oxododecanoyl)-L-homoserine lactone (3O-C12-HSL), can act directly on host TJs. Exposure to 3O-C12-HSL induces a decrease in the cellular contents and tyrosine-hyperphosphorylation of ZO-1, ZO-3 and JAM-A, and simultaneously triggers occludin dephosphorylation (Vikström et al., 2009, 2010). These modifications lead to a loss of interaction between TJ components, resulting in the transient loss of TJ sealing capacity (Vikström et al., 2009; Halldorsson et al., 2010; Schwarzer et al., 2014). Incubation of epithelial cells with 3O-C12-HSL also decreases cellular levels of $E$-cadherin/ $\beta$-catenin and modifies their phosphorylation status, diminishing homophilic binding (Vikström et al., 2009).

\section{The Type III Secretion System}

As mentioned above, $P$. aeruginosa's T3SS delivers exotoxins to the cytoplasm of host cells (Deng and Barbieri, 2008; Hauser, 2009). Four toxins have been identified: ExoU, ExoS, ExoT, and ExoY. In general, the different genes coding for exotoxins are not 


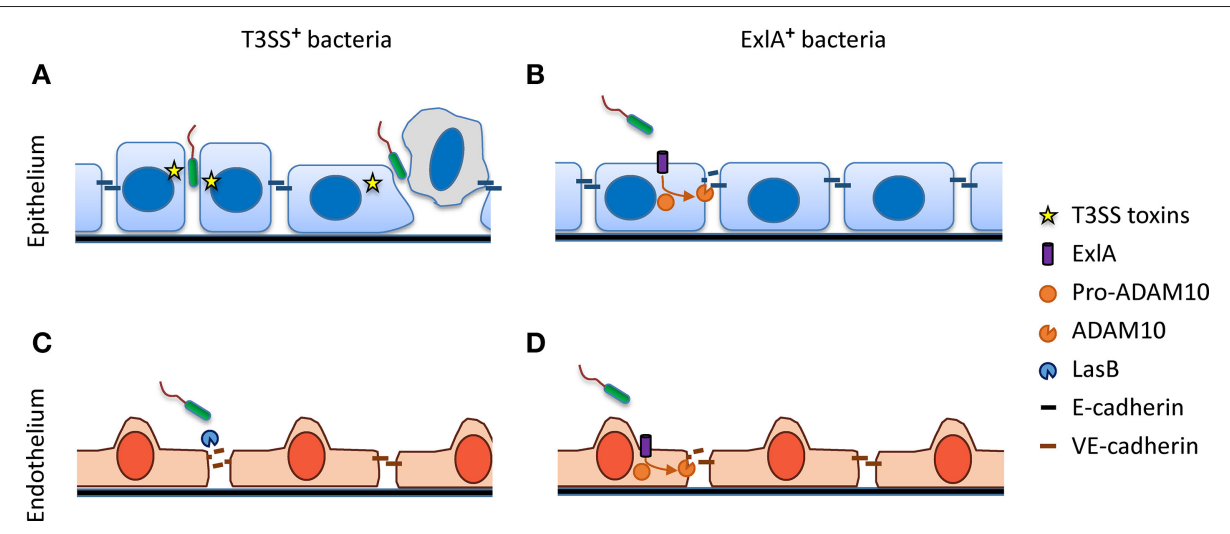

FIGURE 2 | Disruption of adherens junctions by P. aeruginosa. (A) T3SS+ bacteria exploit transient junctional weakenings, occurring during cell division or senecent cell exclusion, to penetrate the epithelial junction cleft. (B) Pore formation induced by ExIA+ bacteria activates the metalloprotease ADAM10, which in turn cleaves E-cadherin. (C) Most T3SS+ bacteria secrete the protease LasB, which cleaves endothelial VE-cadherin. (D) Similar to epithelial cells, ExIA induces VE-cadherin cleavage via ADAM10 activation in endothelial cells.

all present in a single bacterial clone; in particular, exoU and exoS are mutually exclusive. ExoU is a phospholipase inducing plasma membrane disruption and necrotic cell death. Therefore, it promotes bacterial transmigration by killing epithelial cells. ExoY displays nucleotidyl cyclase activity resulting in microtubule and actin fiber alterations, but its toxic activity remains elusive. ExoS and $\mathrm{T}$, present in most hospital strains, have been the subjects of more extensive study. These two toxins display GTPase-activating protein activity, targeting Ras-, Rhoand Rab-family GTPases through their ADP-ribosyl-transferase activity (ExoS) or the focal contact proteins Crk1-2 (ExoT). In particular, inactivation of Rho GTPases (i.e., Rho, Rac and cdc42) by ExoS or ExoT induces the dephosphorylation of Lim kinase and subsequently of cofilin (Huber et al., 2014). Cofilin is an actin depolymerizing enzyme which is active in its unphosphorylated state. Hence, ExoS and ExoT, via inactivation of Rho GTPases, cause actin cytoskeleton depolymerization in all intracellular locations, depriving AJs and TJs of an intracellular component essential for their full adhesive capacity. Rho and Rac are also required at AJs to promote protein complex association and strong cell-cell interaction (Yamada and Nelson, 2007). The inhibition of Rho and Rac by ExoS/ExoT may thus directly affect the organization of the AJ complex. Interestingly, the pharmacological activation of another GTPase, Rap1, which also drives AJ complex organization, was shown to counteract the effect of ExoS/ExoT on junction disruption and to reduce exotoxin-induced cell rounding (Bouillot et al., 2015). In addition, ExoS has been shown to use unexplored mechanisms to displace ZO-1 and occludin from the TJ (Soong et al., 2008).

\section{Type IV Pili}

Toxin injection by the T3SS requires the presence of pili. Tension in the pilus induced by surface attachment triggers the synthesis of various virulence factors, including the T3SS (Persat et al., 2015; Inclan et al., 2016). Pili are also essential for the close bacterium-host cell interaction required for T3SS toxin injection
(Heiniger et al., 2010; Hayashi et al., 2015). Pili thus have a major impact on bacterial transmigration (Heiniger et al., 2010; Hayashi et al., 2015; Golovkine et al., 2016a) in addition to their motility-inducing capacity, and probably each of their functions are essential to this process.

Together, the T3SS, the pili and the flagellum are all required for transmigration across epithelial cell junctions (Heiniger et al., 2010; Hayashi et al., 2015; Golovkine et al., 2016a). However, the T3SS effectors are only injected into host cells through their baso-lateral membrane domain, i.e., when bacteria penetrate the cell layer or when they are in the sub-epithelial compartment (Fleiszig et al., 1997; Lee et al., 1999; Kazmierczak et al., 2004). As a consequence, the T3SS is probably not the primary virulence factor required for bacterial transmigration across mucosae. It is nevertheless actively involved in acute infections, as elegantly shown in infected mouse lungs (Rangel et al., 2015).

\section{ExIA}

Recently, a pore-forming toxin named Exolysin, or ExlA, was identified in a subset of $P$. aeruginosa outlier strains lacking T3SS (Elsen et al., 2014; Huber et al., 2016; Reboud et al., 2016). ExlA is a cytolysin, secreted by a two-partner secretion (TPS) system, that forms a $1.6-\mathrm{nm}$ diameter pore in the host plasma membrane (Basso et al., 2017). ExlA induces E-cadherin cleavage by a multistep, subtly controlled mechanism (Figure 2B) (Reboud et al., 2017). The cadherins are natural substrates of the transmembrane metalloprotease ADAM10 (Pruessmeyer and Ludwig, 2009). Its precursor form, pro-ADAM10, is maintained in an inactive state through interaction with calmodulin, a cytosolic protein which has a high affinity for $\mathrm{Ca}^{2+}$. ExlA pore formation triggers a massive $\mathrm{Ca}^{2+}$ influx into the cytosol, which results in the dissociation of calmodulin and proADAM10. ADAM10 can then be activated through cleavage by furin. In turn, E-cadherin is rapidly cleaved by mature ADAM10, provoking junction disruption (Reboud et al., 2017). Thus, similar to $\alpha$-hemolysin (Hla), a pore-forming toxin from Staphylococcus aureus (Inoshima et al., 2011), ExlA can subvert a 
TABLE 1 | P. aeruginosa virulence factors altering intercellular junctions.

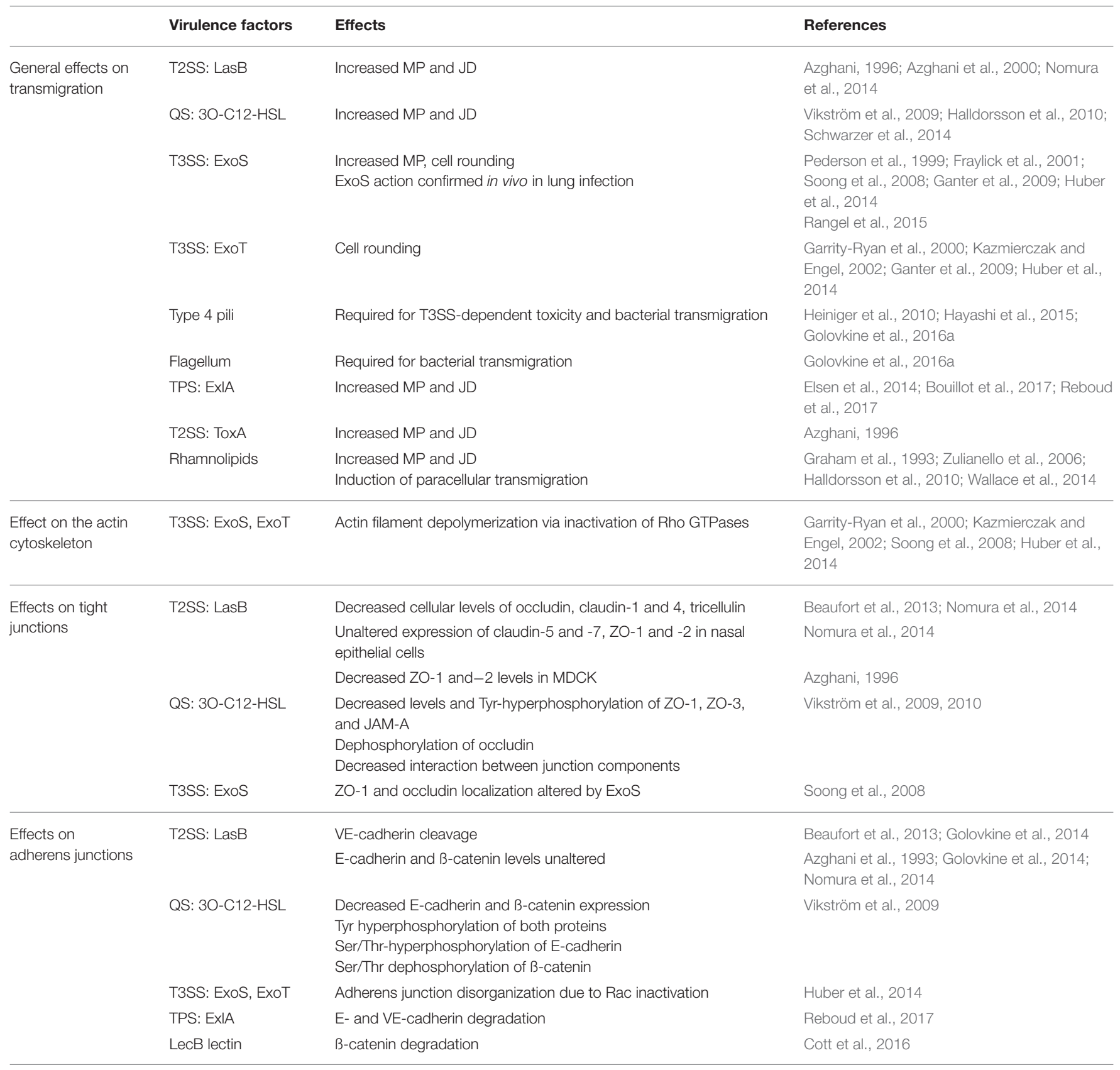

30-C12-HSL, N-(3-oxododecanoyl)-L-homoserine lactone; JD, junction disruption; MP, monolayer permeability; QS, quorum sensing; T2SS, type II secretion system; T3SS, type III secretion system; TPS, two-partner secretion.

highly regulated host mechanism involved in cadherin shedding to efficiently disrupt cell-cell junctions.

\section{Other Factors}

The lectin LecB, located at the surface of $P$. aeruginosa, also targets epithelial AJs, inducing $\beta$-catenin degradation by unknown mechanisms (Cott et al., 2016). The exotoxin A (ToxA or ETA), secreted by the T2SS, inhibits host protein synthesis by ADP-ribosylating elongation factor-2 (Bleves et al., 2010). Exposure to ToxA results in increased monolayer permeability and junction disruption, possibly due to interruption of protein translation (Azghani, 1996). Finally, the rhamnolipids produced by $P$. aeruginosa also appear to be important in the transmigration process, but their mechanisms of action remain to be elucidated (Graham et al., 1993; Zulianello et al., 2006; Halldorsson et al., 2010; Wallace et al., 2014).

From this overview, it emerges clearly that $P$. aeruginosa has developed multiple, highly-targeted virulence strategies affecting the TJs and the AJs to achieve transmigration. 


\section{THE SPECIFIC CASE OF THE VASCULAR ENDOTHELIUM}

The endothelium is the innermost tunica of all vessel types. It is composed of a single thin layer of endothelial cells with cobblestone morphology, and it is endowed with the barrier properties of the vascular system. $P$. aeruginosa transmigration across the endothelium has been investigated in a small number of studies (Ganter et al., 2009; Vasil et al., 2009; Elsen et al., 2014; Golovkine et al., 2014; Huber et al., 2014). Like for epithelial cells, the apical domain of endothelial cells is refractory to injection with the T3SS, whereas their baso-lateral domain is permissive. The intercellular junctions are organized similarly to epithelial junctions, except that no desmosome is present and AJs and TJs are intermingled (Wallez and Huber, 2008). AJs contain an endothelial-specific cadherin, VE-cadherin, and TJs exhibit a different group of claudins. In contrast to E-cadherin, VE-cadherin is sensitive to LasB protease, which cleaves the protein within its extracellular part to release the adhesive domain (Figure 2C) (Beaufort et al., 2013; Golovkine et al., 2014). Thus, LasB is probably P. aeruginosa's master virulence factor, orchestrating its transmigration across endothelia. Once bacteria are present in the intercellular space or underneath the cell, the T3SS and its effectors can effectively induce cellular retraction by dismantling the actin cytoskeleton, further disrupting the endothelial barrier. Like in epithelial cells, VE-cadherin is rapidly cleaved by ExlA-secreting bacteria through a mechanism involving ADAM10 (Figure 2D) (Reboud et al., 2017).

\section{BREACHING THE BASEMENT MEMBRANE}

The basement membrane is a layer of extracellular matrix synthesized by epithelial and endothelial cells. This membrane, composed of laminins and type IV collagen, is in direct contact with the basal side of these cells. Both LasB and AprA, another protease secreted by $P$. aeruginosa, can degrade laminins (Heck et al., 1986a), whereas only LasB efficiently cleaves type IV collagen (Heck et al., 1986b; Bejarano et al., 1989). P. aeruginosa is thus equipped to readily digest and cross this layer. Other matrix proteins found in connective tissues, including type III collagen, fibronectin, vitronectin and elastin can also be cleaved by $P$. aeruginosa proteases (Heck et al., 1986b; Beaufort et al., 2011; Reboud et al., 2016) to allow bacterial progression in the mesenchymal compartment.

\section{CONCLUSION AND FUTURE PROSPECTS}

A number of molecular mechanisms have been reported to explain how $P$. aeruginosa transmigrates across tissue barriers, and a multistep process can be proposed involving the cooperation of several virulence factors at different stages during bacterial penetration. However, these mechanisms were determined in cellular models of infection, and very few in vivo confirmations are available, except for the presence of host protein degradation products and the injection of exotoxins into cells within host tissues. In vivo bacterial transmigration may be influenced by tissue architecture, fluid flow and the immune system. The effects of these factors cannot be fully or easily modeled through reductionist approaches. Intravital microscopy can now be used in lungs, and this approach will be instrumental in helping us to understand how acute infection proceeds in this organ (Masedunskas et al., 2012). In particular, it should provide a better appreciation of the priming steps leading to barrier permissivity. Furthermore, the possibility to observe bacteria by intravascular microscopy (Broadley et al., 2016) should provide information on the fate and extravasation of $P$. aeruginosa in bacteremia.

\section{AUTHOR CONTRIBUTIONS}

All authors contributed to the writing of various parts of the review. PH: made Table 1 and Figure 1; GG: made Figure 2; All authors reviewed the manuscript, the table and the figures.

\section{FUNDING}

The work of the team is supported by grants from Laboratoire of Excellence GRAL (ANR-10-LABX-49-01), Agence Nationale de la Recherche (ANR-15-CE11-0018-01) and Fondation pour la Recherche Médicale “Equipe FRM 2017” (DEQ20170336705). We further acknowledge supports from INSERM, CEA and University Grenoble-Alpes.

\section{REFERENCES}

Azghani, A. O. (1996). Pseudomonas aeruginosa and epithelial permeability: role of virulence factors elastase and exotoxin A. Am. J. Respir. Cell Mol. Biol. 15, 132-140. doi: 10.1165/ajrcmb.15.1.8679217

Azghani, A. O., Gray, L. D., and Johnson, A. R. (1993). A bacterial protease perturbs the paracellular barrier function of transporting epithelial monolayers in culture. Infect. Immun. 61, 2681-2686.

Azghani, A. O., Miller, E. J., and Peterson, B. T. (2000). Virulence factors from Pseudomonas aeruginosa increase lung epithelial permeability. Lung 178, 261-269. doi: 10.1007/s004080000031

Basso, P., Ragno, M., Elsen, S., Reboud, E., Golovkine, G., Bouillot, S., et al. (2017). Pseudomonas aeruginosa pore-forming exolysin and type IV pili cooperate to induce host cell lysis. MBio 8:e2250-16. doi: 10.1128/mBio.02250-16

Beaufort, N., Corvazier, E., Hervieu, A., Choqueux, C., Dussiot, M., Louedec, L., et al. (2011). The thermolysin-like metalloproteinase and virulence factor LasB from pathogenic Pseudomonas aeruginosa induces anoikis of human vascular cells. Cell. Microbiol. 13, 1149-1167. doi: 10.1111/j.1462-5822.2011. 01606.x

Beaufort, N., Corvazier, E., Mlanaoindrou, S., de Bentzmann, S., and Pidard, D. (2013). Disruption of the endothelial barrier by proteases from the bacterial pathogen Pseudomonas aeruginosa: implication of matrilysis and receptor cleavage. PLoS ONE 8:e75708. doi: 10.1371/journal.pone.0075708

Bejarano, P. A., Langeveld, J. P., Hudson, B. G., and Noelken, M. E. (1989). Degradation of basement membranes by Pseudomonas aeruginosa elastase. Infect. Immun. 57, 3783-3787.

Bleves, S., Viarre, V., Salacha, R., Michel, G. P., Filloux, A., and Voulhoux, R. (2010). Protein secretion systems in Pseudomonas aeruginosa: a 
wealth of pathogenic weapons. Int. J. Med. Microbiol. 300, 534-543. doi: 10.1016/j.ijmm.2010.08.005

Boettner, B., and Van Aelst, L. (2009). Control of cell adhesion dynamics by Rap1 signaling. Curr. Opin. Cell Biol. 21, 684-693. doi: 10.1016/j.ceb.2009.06.004

Bouillot, S., Attrée, I., and Huber, P. (2015). Pharmacological activation of Rap1 antagonizes the endothelial barrier disruption induced by exotoxins ExoS and ExoT of Pseudomonas aeruginosa. Infect. Immun. 83, 1820-1829. doi: 10.1128/IAI.00010-15

Bouillot, S., Munro, P., Gallet, B., Reboud, E., Cretin, F., Golovkine, G., et al. (2017). Pseudomonas aeruginosa exolysin promotes bacterial growth in lungs, alveolar damage and bacterial dissemination. Sci. Rep. 7:2120. doi: 10.1038/s41598-017-02349-0

Broadley, S. P., Plaumann, A., Coletti, R., Lehmann, C., Wanisch, A., Seidlmeier, A., et al. (2016). Dual-track clearance of circulating bacteria balances rapid restoration of blood sterility with induction of adaptive immunity. Cell Host Microbe 20, 36-48. doi: 10.1016/j.chom.2016.05.023

Cereijido, M., Contreras, R. G., and Shoshani, L. (2004). Cell adhesion, polarity, and epithelia in the dawn of metazoans. Physiol. Rev. 84, 1229-1262. doi: 10.1152/physrev.00001.2004

Clark, C. A., Thomas, L. K., and Azghani, A. O. (2011). Inhibition of protein kinase C attenuates Pseudomonas aeruginosa elastase-induced epithelial barrier disruption. Am. J. Respir. Cell Mol. Biol. 45, 1263-1271. doi: $10.1165 / \mathrm{rcmb} .2010-04590 \mathrm{C}$

Cott, C., Thuenauer, R., Landi, A., Kühn, K., Juillot, S., Imberty, A., et al. (2016). Pseudomonas aeruginosa lectin LecB inhibits tissue repair processes by triggering beta-catenin degradation. Biochim. Biophys. Acta 1863(6 Pt A), 1106-1118. doi: 10.1016/j.bbamcr.2016.02.004

Coureuil, M., Mikaty, G., Miller, F., Lécuyer, H., Bernard, C., Bourdoulous, S., et al. (2009). Meningococcal type IV pili recruit the polarity complex to cross the brain endothelium. Science 325, 83-87. doi: 10.1126/science.1173196

Deng, Q., and Barbieri, J. T. (2008). Molecular mechanisms of the cytotoxicity of ADP-ribosylating toxins. Annu. Rev. Microbiol. 62, 271-288. doi: 10.1146/annurev.micro.62.081307.162848

Doran, K. S., Banerjee, A., Disson, O., and Lecuit, M. (2013). Concepts and mechanisms: crossing host barriers. Cold Spring Harb Perspect Med. 3:a010090. doi: 10.1101/cshperspect.a010090

Elsen, S., Huber, P., Bouillot, S., Couté, Y., Fournier, P., Dubois, Y., et al. (2014). A type III secretion negative clinical strain of Pseudomonas aeruginosa employs a two-partner secreted exolysin to induce hemorrhagic pneumonia. Cell Host Microbe 15, 164-176. doi: 10.1016/j.chom.2014.01.003

Farquhar, M. G., and Palade, G. E. (1963). Junctional complexes in various epithelia. J. Cell Biol. 17, 375-412. doi: 10.1083/jcb.17.2.375

Fleiszig, S. M., and Evans, D. J. (2010). Pathogenesis of contact lens-associated microbial keratitis. Optom. Vis. Sci. 87, 225-232. doi: 10.1097/OPX.0b013e3181eeddf9

Fleiszig, S. M., Evans, D. J., Do, N., Vallas, V., Shin, S., and Mostov, K. E. (1997). Epithelial cell polarity affects susceptibility to Pseudomonas aeruginosa invasion and cytotoxicity. Infect. Immun. 65, 2861-2867.

Fleiszig, S. M., Zaidi, T. S., Fletcher, E. L., Preston, M. J., and Pier, G. B. (1994). Pseudomonas aeruginosa invades corneal epithelial cells during experimental infection. Infect. Immun. 62, 3485-3493.

Fraylick, J. E., La Rocque, J. R., Vincent, T. S., and Olson, J. C. (2001). Independent and coordinate effects of ADP-ribosyltransferase and GTPaseactivating activities of exoenzyme S on HT-29 epithelial cell function. Infect. Immun. 69, 5318-5328. doi: 10.1128/IAI.69.9.5318-5328.2001

Ganter, M. T., Roux, J., Su, G., Lynch, S. V., Deutschman, C. S., Weiss, Y. G., et al. (2009). Role of small GTPases and alphavbeta5 integrin in Pseudomonas aeruginosa-induced increase in lung endothelial permeability. Am. J. Respir. Cell Mol. Biol. 40, 108-118. doi: 10.1165/rcmb.2007-0454OC

Garrity-Ryan, L., Kazmierczak, B., Kowal, R., Comolli, J., Hauser, A., and Engel, J. N. (2000). The arginine finger domain of ExoT contributes to actin cytoskeleton disruption and inhibition of internalization of Pseudomonas aeruginosa by epithelial cells and macrophages. Infect. Immun. 68, 7100-7113. doi: 10.1128/IAI.68.12.7100-7113.2000

Gellatly, S. L., and Hancock, R. E. (2013). Pseudomonas aeruginosa: new insights into pathogenesis and host defenses. Pathog. Dis. 67, 159-173. doi: 10.1111/2049-632X.12033
Golovkine, G., Faudry, E., Bouillot, S., Elsen, S., Attrée, I., and Huber, P. (2016a). Pseudomonas aeruginosa transmigrates at epithelial cell-cell junctions, exploiting sites of cell division and senescent cell extrusion. PLoS Pathog. 12:e1005377. doi: 10.1371/journal.ppat.1005377

Golovkine, G., Faudry, E., Bouillot, S., Voulhoux, R., Attrée, I., and Huber, P. (2014). VE-cadherin cleavage by LasB protease from Pseudomonas aeruginosa facilitates type III secretion system toxicity in endothelial cells. PLoS Pathog. 10:e1003939. doi: 10.1371/journal.ppat.1003939

Golovkine, G., Lemelle, L., Burny, C., Vaillant, C., Palierne, J. F., Place, C., et al. (2016b). Host cell surfaces induce a type IV pili-dependent alteration of bacterial swimming. Sci. Rep. 6:38950. doi: 10.1038/srep38950

Graham, A., Steel, D. M., Wilson, R., Cole, P. J., Alton, E. W., and Geddes, D. M. (1993). Effects of purified Pseudomonas rhamnolipids on bioelectric properties of sheep tracheal epithelium. Exp. Lung Res. 19, 77-89. doi: 10.3109/01902149309071082

Halldorsson, S., Gudjonsson, T., Gottfredsson, M., Singh, P. K., Gudmundsson, G. H., and Baldursson, O. (2010). Azithromycin maintains airway epithelial integrity during Pseudomonas aeruginosa infection. Am. J. Respir. Cell Mol. Biol. 42, 62-68. doi: 10.1165/rcmb.2008-0357OC

Hauser, A. R. (2009). The type III secretion system of Pseudomonas aeruginosa: infection by injection. Nat. Rev. 7, 654-665. doi: 10.1038/nrmicro2199

Hauser, A. R., Fleiszig, S., Kang, P. J., Mostov, K., and Engel, J. N. (1998). Defects in type III secretion correlate with internalization of Pseudomonas aeruginosa by epithelial cells. Infect. Immun. 66, 1413-1420.

Hayashi, N., Nishizawa, H., Kitao, S., Deguchi, S., Nakamura, T., Fujimoto, A., et al. (2015). Pseudomonas aeruginosa injects type III effector ExoS into epithelial cells through the function of type IV pili. FEBS Lett. 589, 890-896. doi: 10.1016/j.febslet.2015.02.031

Heck, L. W., Morihara, K., and Abrahamson, D. R. (1986a). Degradation of soluble laminin and depletion of tissue-associated basement membrane laminin by Pseudomonas aeruginosa elastase and alkaline protease. Infect. Immun. 54, 149-153.

Heck, L. W., Morihara, K., McRae, W. B., and Miller, E. J. (1986b). Specific cleavage of human type III and IV collagens by Pseudomonas aeruginosa elastase. Infect. Immun. 51, 115-118.

Heiniger, R. W., Winther-Larsen, H. C., Pickles, R. J., Koomey, M., and Wolfgang, M. C. (2010). Infection of human mucosal tissue by Pseudomonas aeruginosa requires sequential and mutually dependent virulence factors and a novel pilus-associated adhesin. Cell. Microbiol. 12, 1158-1173. doi: 10.1111/j.1462-5822.2010.01461.x

Huber, P., Basso, P., Reboud, E., and Attrée, I. (2016). Pseudomonas aeruginosa renews its virulence factors. Environ. Microbiol. Rep. 8, 564-571. doi: 10.1111/1758-2229.12443

Huber, P., Bouillot, S., Elsen, S., and Attrée, I. (2014). Sequential inactivation of Rho GTPases and Lim kinase by Pseudomonas aeruginosa toxins ExoS and ExoT leads to endothelial monolayer breakdown. Cell. Mol. Life Sci. 71, 1927-1941. doi: 10.1007/s00018-013-1451-9

Inclan, Y. F., Persat, A., Greninger, A., Von Dollen, J., Johnson, J., Krogan, N., et al. (2016). A scaffold protein connects type IV pili with the Chp chemosensory system to mediate activation of virulence signaling in Pseudomonas aeruginosa. Mol. Microbiol. 101, 590-605. doi: 10.1111/mmi.13410

Inoshima, I., Inoshima, N., Wilke, G. A., Powers, M. E., Frank, K. M., Wang, Y., et al. (2011). A Staphylococcus aureus pore-forming toxin subverts the activity of ADAM10 to cause lethal infection in mice. Nat. Med. 17, 1310-1314. doi: $10.1038 / \mathrm{nm} .2451$

Kazmierczak, B. I., and Engel, J. N. (2002). Pseudomonas aeruginosa ExoT acts in vivo as a GTPase-activating protein for RhoA, Racl, and Cdc42. Infect. Immun. 70, 2198-2205. doi: 10.1128/IAI.70.4.2198-2205.2002

Kazmierczak, B. I., Mostov, K., and Engel, J. N. (2004). Epithelial cell polarity alters Rho-GTPase responses to Pseudomonas aeruginosa. Mol. Biol. Cell 15, 411-419. doi: 10.1091/mbc.E03-08-0559

Kierbel, A., Gassama-Diagne, A., Mostov, K., and Engel, J. N. (2005). The phosphoinositol-3-kinase-protein kinase B/Akt pathway is critical for Pseudomonas aeruginosa strain PAK internalization. Mol. Biol. Cell 16, 2577-2585. doi: 10.1091/mbc.E04-08-0717

Kierbel, A., Gassama-Diagne, A., Rocha, C., Radoshevich, L., Olson, J., Mostov, K., et al. (2007). Pseudomonas aeruginosa exploits a PIP3-dependent pathway 
to transform apical into basolateral membrane. J. Cell Biol. 177, 21-27. doi: $10.1083 /$ jcb. 200605142

Lee, A., Chow, D., Haus, B., Tseng, W., Evans, D., Fleiszig, S., et al. (1999). Airway epithelial tight junctions and binding and cytotoxicity of Pseudomonas aeruginosa. Am. J. Physiol. 277(1 Pt 1), L204-L217.

Leighton, T. L., Buensuceso, R. N., Howell, P. L., and Burrows, L. L. (2015). Biogenesis of Pseudomonas aeruginosa type IV pili and regulation of their function. Environ. Microbiol. 17, 4148-4163. doi: 10.1111/1462-2920.12849

Lepanto, P., Bryant, D. M., Rossello, J., Datta, A., Mostov, K. E., and Kierbel, A. (2011). Pseudomonas aeruginosa interacts with epithelial cells rapidly forming aggregates that are internalized by a Lyn-dependent mechanism. Cell. Microbiol. 13, 1212-1222. doi: 10.1111/j.1462-5822.2011.01611.x

Luzar, M. A., Thomassen, M. J., and Montie, T. C. (1985). Flagella and motility alterations in Pseudomonas aeruginosa strains from patients with cystic fibrosis: relationship to patient clinical condition. Infect. Immun. 50, 577-582.

Lyczak, J. B., Cannon, C. L., and Pier, G. B. (2000). Establishment of Pseudomonas aeruginosa infection: lessons from a versatile opportunist. Microbes Infect. 2, 1051-1060. doi: 10.1016/S1286-4579(00)01259-4

Masedunskas, A., Milberg, O., Porat-Shliom, N., Sramkova, M., Wigand, T., Amornphimoltham, P., et al. (2012). Intravital microscopy: a practical guide on imaging intracellular structures in live animals. Bioarchitecture 2, 143-157. doi: $10.4161 /$ bioa. 21758

McCole, D. F. (2013). Phosphatase regulation of intercellular junctions. Tissue Barriers 1:e26713. doi: 10.4161/tisb.26713

Mittal, R., Aggarwal, S., Sharma, S., Chhibber, S., and Harjai, K. (2009). Urinary tract infections caused by Pseudomonas aeruginosa: a minireview. J. Infect. Public Health 2, 101-111. doi: 10.1016/j.jiph.2009.08.003

Nomura, K., Obata, K., Keira, T., Miyata, R., Hirakawa, S., Takano, K., et al. (2014). Pseudomonas aeruginosa elastase causes transient disruption of tight junctions and downregulation of PAR-2 in human nasal epithelial cells. Respir. Res. 15:21. doi: 10.1186/1465-9921-15-21

Pederson, K. J., Vallis, A. J., Aktories, K., Frank, D. W., and Barbieri, J. T. (1999). The amino-terminal domain of Pseudomonas aeruginosa ExoS disrupts actin filaments via small-molecular-weight GTP-binding proteins. Mol. Microbiol. 32, 393-401. doi: 10.1046/j.1365-2958.1999.01359.x

Persat, A., Inclan, Y. F., Engel, J. N., Stone, H. A., and Gitai, Z. (2015). Type IV pili mechanochemically regulate virulence factors in Pseudomonas aeruginosa. Proc. Natl. Acad. Sci. U.S.A. 112, 7563-7568. doi: 10.1073/pnas.1502025112

Pruessmeyer, J., and Ludwig, A. (2009). The good, the bad and the ugly substrates for ADAM10 and ADAM17 in brain pathology, inflammation and cancer. Semin. Cell Dev. Biol. 20, 164-174. doi: 10.1016/j.semcdb.2008.09.005

Rangel, S. M., Diaz, M. H., Knoten, C. A., Zhang, A., and Hauser, A. R. (2015). The Role of ExoS in Dissemination of Pseudomonas aeruginosa during Pneumonia. PLoS Pathog. 11:e1004945. doi: 10.1371/journal.ppat.1004945

Ratheesh, A., Priya, R., and Yap, A., (2013). Coordinating and Rac: the regulation of Rho GTPase signaling and cadherin junctions. Prog. Mol. Biol. Transl. Sci. 116, 49-68. doi: 10.1016/B978-0-12-394311-8.00003-0

Reboud, E., Bouillot, S., Patot, S., Béganton, B., Attrée, I., and Huber, P. (2017). Pseudomonas aeruginosa ExlA and Serratia marcescens ShlA trigger cadherin cleavage by promoting calcium influx and ADAM10 activation. PLoS Pathog. 13:e1006579. doi: 10.1371/journal.ppat.1006579

Reboud, E., Elsen, S., Bouillot, S., Golovkine, G., Basso, P., Jeannot, K., et al. (2016). Phenotype and toxicity of the recently discovered exlA-positive Pseudomonas aeruginosa strains collected worldwide. Environ. Microbiol. 18, 3425-3439. doi: $10.1111 / 1462-2920.13262$

Sana, T. G., Baumann, C., Merdes, A., Soscia, C., Rattei, T., Hachani, A., et al. (2015). Internalization of Pseudomonas aeruginosa Strain PAO1 into epithelial cells is promoted by interaction of a T6SS effector with the microtubule network. MBio 6:e00712-15. doi: 10.1128/mBio.00712-15

Sawa, T. (2014). The molecular mechanism of acute lung injury caused by Pseudomonas aeruginosa: from bacterial pathogenesis to host response. J. Intensive Care 2:10. doi: 10.1186/2052-0492-2-10

Schuster, M., Lostroh, C. P., Ogi, T., and Greenberg, E. P. (2003). Identification timing, and signal specificity of Pseudomonas aeruginosa quorum- controlled genes: a transcriptome analysis. J. Bacteriol. 185, 2066-2079. doi: 10.1128/JB.185.7.2066-2079.2003

Schwarzer, C., Ravishankar, B., Patanwala, M., Shuai, S., Fu, Z., Illek, B., et al. (2014). Thapsigargin blocks Pseudomonas aeruginosa homoserine lactoneinduced apoptosis in airway epithelia. Am. J. Physiol. Cell Physiol. 306, C844C855. doi: 10.1152/ajpcell.00002.2014

Serrels, A., Canel, M., Brunton, V. G., and Frame, M. C. (2011). Src/FAKmediated regulation of E-cadherin as a mechanism for controlling collective cell movement: insights from in vivo imaging. Cell Adh. Migr. 5, 360-365. doi: 10.4161/cam.5.4.17290

Soong, G., Parker, D., Magargee, M., and Prince, A. S. (2008). The type III toxins of Pseudomonas aeruginosa disrupt epithelial barrier function. J. Bacteriol. 190, 2814-2821. doi: 10.1128/JB.01567-07

Tran, C. S., Eran, Y., Ruch, T. R., Bryant, D. M., Datta, A., Brakeman, P., et al. (2014). Host cell polarity proteins participate in innate immunity to Pseudomonas aeruginosa infection. Cell Host Microbe 15, 636-643. doi: 10.1016/j.chom.2014.04.007

Turner, K. H., Everett, J., Trivedi, U., Rumbaugh, K. P., and Whiteley, M. (2014). Requirements for Pseudomonas aeruginosa acute burn and chronic surgical wound infection. PLoS Genet. 10:e1004518. doi: 10.1371/journal.pgen. 1004518

Vasil, M. L., Stonehouse, M. J., Vasil, A. I., Wadsworth, S. J., Goldfine, H., Bolcome, R. E. III., et al. (2009). A complex extracellular sphingomyelinase of Pseudomonas aeruginosa inhibits angiogenesis by selective cytotoxicity to endothelial cells. PLoS Pathog. 5:e1000420. doi: 10.1371/journal.ppat.1000420

Vikström, E., Bui, L., Konradsson, P., and Magnusson, K. E. (2009). The junctional integrity of epithelial cells is modulated by Pseudomonas aeruginosa quorum sensing molecule through phosphorylation-dependent mechanisms. Exp. Cell Res. 315, 313-326. doi: 10.1016/j.yexcr.2008.10.044

Vikström, E., Bui, L., Konradsson, P., and Magnusson, K. E. (2010). Role of calcium signalling and phosphorylations in disruption of the epithelial junctions by Pseudomonas aeruginosa quorum sensing molecule. Eur. J. Cell Biol. 89, 584-597. doi: 10.1016/j.ejcb.2010.03.002

Wallace, C. J., Medina, S. H., and ElSayed, M. E. (2014). Effect of rhamnolipids on permeability across Caco-2 cell monolayers. Pharm. Res. 31, 887-894. doi: $10.1007 / \mathrm{s} 11095-013-1210-5$

Wallez, Y., and Huber, P. (2008). Endothelial adherens and tight junctions in vascular homeostasis, inflammation and angiogenesis. Biochim. Biophys. Acta 1778, 794-809. doi: 10.1016/j.bbamem.2007.09.003

Williams, B. J., Dehnbostel, J., and Blackwell, T. S. (2010). Pseudomonas aeruginosa: host defence in lung diseases. Respirology 15, 1037-1056. doi: 10.1111/j.1440-1843.2010.01819.x

Yamada, S., and Nelson, W. J. (2007). Localized zones of Rho and Rac activities drive initiation and expansion of epithelial cell-cell adhesion. J. Cell Biol. 178, 517-527. doi: 10.1083/jcb.200701058

Zaas, D. W., Swan, Z. D., Brown, B. J., Li, G., Randell, S. H., Degan, S., et al. (2009). Counteracting signaling activities in lipid rafts associated with the invasion of lung epithelial cells by Pseudomonas aeruginosa. J. Biol. Chem. 284, 9955-9964. doi: 10.1074/jbc.M808629200

Zulianello, L., Canard, C., Köhler, T., Caille, D., Lacroix, J. S., and Meda, P. (2006). Rhamnolipids are virulence factors that promote early infiltration of primary human airway epithelia by Pseudomonas aeruginosa. Infect. Immun. 74, 3134-3147. doi: 10.1128/IAI.01772-05

Conflict of Interest Statement: The authors declare that the research was conducted in the absence of any commercial or financial relationships that could be construed as a potential conflict of interest.

Copyright (c) 2018 Golovkine, Reboud and Huber. This is an open-access article distributed under the terms of the Creative Commons Attribution License (CC BY). The use, distribution or reproduction in other forums is permitted, provided the original author(s) or licensor are credited and that the original publication in this journal is cited, in accordance with accepted academic practice. No use, distribution or reproduction is permitted which does not comply with these terms. 